\title{
ANATOMIA DA ARTÉRIA HEPÁTICA EM DOADORES E RECEPTORES DE TRANSPLANTE HEPÁTICO INTERVIVOS
}

\section{HEPATIC ARTERY ANATOMY IN LIVING RELATED LIVER TRANSPLANTATION}

\author{
Renato Vianna Soares ${ }^{1}$; Júlio Cezar U. Coelho, TCBC-PR ${ }^{2}$; Jorge E. F. Matias, TCBC-PR ; \\ Clementino Zeni Neto, TCBC-PR ; Alexandre C.T. de Freitas ${ }^{4}$; José Luis de Godoy ${ }^{4}$
}

\begin{abstract}
RESUMO: Objetivo:O objetivo do presente estudo é avaliar a anatomia da artéria hepática nos doadores e receptores do transplante hepático intervivos realizados no Hospital de Clínicas da Universidade Federal do Paraná e do Hospital Nossa Senhora das Graças de Curitiba. Método: A avaliação foi retrospectiva de março de 1998 até setembro de 2002 (23 transplantes), quando os dados passaram a ser captados de forma prospectiva (17 transplantes), até agosto de 2003. Foram obtidos dados de 80 pacientes consecutivos (40 doadores e 40 receptores), submetidos a transplante hepático intervivos, sendo 32 transplantes com receptor adulto e 8 transplantes pediátricos (receptor com idade inferior a 15 anos). Entre os 80 pacientes incluídos no estudo, 51 eram do sexo masculino ( 27 receptores e 24 doadores) e 29 eram do sexo feminino (13 receptores e 16 doadores). A idade média dos doadores foi de 32,6 anos e a dos receptores de 36,3 anos. Resultados: No estudo da anatomia da artéria hepática, realizaram-se arteriografias em 43 pacientes, e variações anatômicas foram encontradas em 18 casos $(41,86 \%)$, sendo a mais comum a artéria hepática direita ramo da artéria mesentérica superior $(12,5 \% ; \mathrm{n}=5)$; no estudo da anatomia arterial realizado nas cirurgias, foram verificadas variações em 16 casos $(20 \%)$ entre os 80 casos estudados, sendo a mais comum a artéria hepática direita como ramo da artéria mesentérica superior $(7,5 \% ; n=6)$. Conclusão: Conclui-se que a prevalência de variações na anatomia da artéria hepática é elevada (Rev. Col. Bras. Cir. 2006; 33(2): 63-67).
\end{abstract}

Descritores: Transplante de fígado; Doadores vivos; Artéria hepática/anatomia e histologia.

\section{INTRODUÇÃO}

Os bons resultados do transplante hepático como tratamento da insuficiência hepática terminal vêm ampliando as indicações do transplante, porém a escassez de enxertos é o principal fator limitante para o aumento da realização de transplantes de fígado. Para diminuir esta escassez, técnicas inovadoras como a divisão hepática e o transplante hepático com doadores vivos vêm sendo utilizadas. O primeiro transplante intervivos foi realizado por Raia et al. em $1989^{1}$, e em 1990 Strong et al. $^{2}$ realizaram o primeiro transplante hepático intervivos com êxito. Hashikura et al. ${ }^{3}$ realizaram em 1994 o primeiro transplante intervivos entre adultos, utilizando o lobo esquerdo do fígado. Inicialmente, o transplante intervivos era indicado para crianças e adultos em condições graves e em países onde o número de doadores cadáveres era pequeno ou inexistente. Em nosso meio, as técnicas cirúrgicas nos transplantes com doadores vivos foram divulgadas por Carone $e t$ al. em $1997^{4}$.

Variações anatômicas nos elementos vasculares hepáticos são freqüentes e seu reconhecimento e correto manejo são críticos para o sucesso do transplante hepático intervivos. Um número expressivo de complicações nos trans- plantes intervivos é devido à falhas no reconhecimento de variações anatômicas 5 . Em relação à artéria hepática, variações ocorrem em aproximadamente em $45 \%$ da população e podem influenciar a captação e o implante nos transplantes hepáticos intervivos ${ }^{6}$. O objetivo do presente estudo foi avaliar a anatomia da artéria hepática em doadores e receptores de transplantes intervivos de fígado.

\section{MÉTODO}

A amostra utilizada compreendeu doadores e receptores dos transplantes hepáticos intervivos feitos no Hospital de Clínicas da Universidade Federal do Paraná e no Hospital Nossa Senhora das Graças de Curitiba, durante o período de março de 1998 até agosto de 2003. A avaliação foi retrospectiva de março de 1998 até setembro de 2002 (23 transplantes), quando os dados passaram a ser obtidos de forma prospectiva (17 transplantes).

$\mathrm{Na}$ avaliação retrospectiva foram revisados os protocolos de estudo de doadores e receptores de transplante hepático intervivos. Esses protocolos continham dados dos pacientes, inclusive os de anamnese, de exame físico, de laboratório e de imagem. Observações intra-operatórias da anato-

1 - Mestre em Clínica Cirúrgica pela Universidade Federal do Paraná.

2 - Professor Titular e Chefe dos Serviços de Cirurgia do Aparelho Digestivo e de Transplante Hepático da Universidade Federal do Paraná.

3 - Professor Adjunto e Coordenador do Curso de Pós-Graduação em Clínica Cirúrgica da Universidade Federal do Paraná.

4- Cirurgião do Serviço de Transplante Hepático da Universidade Federal do Paraná.

Recebido em 10/10/2005

Aceito para publicação em 08/12/2005

Conflito de interesses: nenhum

Fonte de Financiamento: nenhuma

Trabalho realizado no serviço de Transplante Hepático da Universidade Federal do Paraná. 
Tabela 1 - Anatomia da artéria hepática - achados de arteriografia.

\begin{tabular}{lrr}
\hline Descrição & N & \% \\
\hline Anatomia normal da artéria hepática & 25 & 58,1 \\
Artéria hepática direita ramo da mesentérica superior & 5 & 12,5 \\
Artéria hepática direita acessória ramo da mesentérica superior & 3 & 7,0 \\
Bifurcação extra-hepática da artéria hepática esquerda & 3 & 7,0 \\
Artéria hepática direita ramo da mesentérica superior com artéria hepática esquerda & & \\
$\quad$ ramo do tronco celíaco & 2 & 4,6 \\
Artéria hepática esquerda ramo do tronco celíaco & 2 & 4,6 \\
Tronco celíacomesentérico* & 2 & 4,6 \\
Artéria hepática comum ramo da artéria mesentérica superior & 1 & 2,3 \\
Total & 43 & 100,0 \\
\hline
\end{tabular}

Nota: Em um dos casos de origem comum do tronco celíaco e da artéria mesentérica superior, a artéria hepática direita foi ramo do tronco celíaco.

mia hepática foram compiladas, principalmente em relação à anatomia da artéria hepática.

$\mathrm{Na}$ avaliação prospectiva as informações dos doadores e receptores foram computadas à medida que os procedimentos foram sendo realizados. Foram também registrados dados em relação ao sexo, idade e sobre a anatomia arterial do fígado obtida de angiografias pré-operatórias. As variações encontradas durante $\mathrm{o}$ ato cirúrgico foram compiladas e documentadas por meio de fotografias. Considerou-se anatomia normal da artéria hepática quando o tronco celíaco com origem na aorta, ramificou-se em artéria gástrica esquerda, artéria esplênica e artéria hepática comum. Esta última, após a emergência da artéria gastroduodenal, continua-se como artéria hepática própria e ramifica-se como artéria hepática direita e esquerda no hilo hepático ${ }^{6-8}$.

As operações para captação dos órgãos compreenderam ressecções dos segmentos II e III de Couinaud para pacientes pediátricos com peso abaixo de $15 \mathrm{Kg}$; ressecções dos segmentos II, III, IV para pacientes pediátricos com maior peso e ressecções dos segmentos V, VI, VII e VIII para o transplante intervivos com receptor adulto.

Em 41 transplantes, foram obtidos dados de 82 pacientes. Foram nove transplantes pediátricos (receptores com idade inferior a 15 anos) e 32 transplantes adultos. Em um caso de transplante hepático tipo "dominó", o receptor do fígado cadavérico foi incluído neste estudo como doador intervivo. Nos casos restantes, os doadores e receptores eram relacionados. Foram excluídos do estudo dois pacientes (um receptor pediátrico e um doador para transplante pediátrico) pois o prontuário não foi localizado. Em dois casos, o transplante foi suspenso em razão de variações venosas significativas no doador, que não foram diagnosticadas no pré-operatório e que poderiam predispor à trombose da veia porta e colocar em risco a vida do doador. Estes dois casos foram incluídos no estudo. Portanto de um total de 82 pacientes, foram incluídos no estudo 80 pacientes: 40 doadores e 40 receptores.

A idade dos pacientes variou entre 8 meses e 67 anos. A média de idade foi de 34,5 anos. Cinqüenta e um pacientes eram do sexo masculino $(63,8 \%)$. A média de idade entre os homens foi de 37,2 anos. Pacientes do sexo feminino somaram 29 casos (36,3\%), com média de idade de 29,7 anos.
Em relação aos 40 doadores, 16 eram do sexo feminino (média de idade de 24,9 anos) e 24 eram do sexo masculino (média de idade de 31,5 anos). A média de idade dos doadores foi de 32,6 anos.

Em relação aos 40 receptores, 27 eram do sexo masculino (média de idade de 41,8 anos) e 13 eram do sexo feminino (média de idade de 24,9 anos). A média de idade dos receptores foi de 36,3 anos.

Foram utilizados três tipos de enxertos: segmentos II e III de Couinaud em cinco casos; segmentos II, III, IV em três casos; e segmentos V, VI, VII e VIII em 32 casos.

Este estudo foi aprovado pela comissão de ética do Hospital de Clínicas da Universidade Federal do Paraná (protocolo CEP/HC 759.178/2003-11).

\section{RESULTADOS}

Variações anatômicas da artéria hepática diagnosticadas por arteriografia

Entre os 80 pacientes do estudo, 43 foram submetidos à arteriografia: 39 entre os 40 doadores e quatro receptores. O único doador que não foi submetido à arteriografia préoperatória foi o do transplante "dominó" (doador de fígado total). Foram encontradas 18 variações na anatomia da artéria hepática $(41,86 \%)$ entre os 43 casos. Nos 25 pacientes restantes $(51,8 \%)$, a anatomia foi normal.

Em ordem decrescente de freqüência, as variações observadas nos exames de arteriografia estão evidenciadas na Tabela 1. As principais variações foram: artéria hepática direita ramo da artéria mesentérica superior em cinco pacientes (12,5\%); artéria hepática direita acessória ramo da artéria mesentérica superior em três casos (7\%); bifurcação extrahepática da artéria hepática esquerda em três pacientes (7\%).

Variações anatômicas da artéria hepática diagnosticadas na operação

Foram descritas variações em 16 pacientes $(20 \%)$ entre os 80 casos estudados. Nos 64 casos restantes (80\%), a anatomia foi considerada normal. Variações foram encontradas em 11 doadores $(27,5 \%)$ entre os 40 estudados. Quanto aos receptores, variações anatômicas foram encontradas em seis casos entre $40(15 \%)$. As variações anatômicas verificadas, 
Tabela 2 - Anatomia da artéria hepática - achados operatórios.

\begin{tabular}{lrr}
\hline Descrição & $\mathbf{N}$ & $\mathbf{\%}$ \\
\hline Normal & 64 & 80,0 \\
Artéria hepática direita ramo da mesentérica superior & 6 & 7,5 \\
Artéria hepática direita ramo acessório da mesentérica superior & 3 & 3,75 \\
Artéria hepática esquerda ramo da gástrica esquerda com hepática direita ramo da mesentérica superior & 2 & 2,5 \\
Artéria hepática esquerda ramo da gástrica esquerda & 1 & 1,25 \\
Artéria hepática esquerda ramo da gástrica esquerda com hepática direita acessória & 1 & 1,25 \\
$\quad$ ramo da mesentérica superior & 1 & 1,25 \\
Artéria hepática direita ramo do tronco celíaco & 1 & 1,25 \\
Artéria hepática comum ramo da mesentérica superior & 1 & 1,25 \\
Ramo extra-hepático da artéria hepática comum direcionado ao setor posterior direito & 80 & 100,0 \\
Total & &
\end{tabular}

em ordem decrescente de freqüência, são mostradas na Tabela 2. As principais variações foram: artéria hepática direita ramo da artéria mesentérica superior (Figura 1) em seis pacientes $(7,5 \%)$; artéria hepática direita acessória ramo da artéria mesentérica superior em três casos $(3,75 \%)$; artéria hepática esquerda ramo da artéria gástrica esquerda com artéria hepática direita ramo da artéria mesentérica superior em dois casos $(2,5 \%)$.

\section{Comparação da anatomia da artéria hepática na arteriografia e na operação}

Efetuando-se uma comparação entre as variações anatômicas da artéria hepática obtidas em exames de arteriografia e as constatadas durante as operações, em 24 pacientes $(55,8 \%)$, houve concordância da arteriografia e dos achados operatórios, quanto à normalidade anatômica da artéria hepática. Nos 19 pacientes restantes, foram encontradas variações na arteriografia, na operação ou em ambas (Tabela 3). Em seis pacientes a arteriografia evidenciou anatomia aberrante que não foi confirmada na operação $(13,95 \%)$. Em um caso, a arteriografia foi normal e na operação verificou-se a existência de um ramo extra-hepático da artéria hepática comum direcionado ao setor posterior direito do fígado.

\section{DISCUSSÃO}

Há várias séries na literatura que descrevem a anatomia da artéria hepática em doadores cadáveres de fígado. Hiatt et al. (1994) encontraram um índice de $24,36 \%$ de variações na artéria hepática, em um estudo com 1000 doadores; Soin et al. $(1996)^{8}$ descreveram 30,60\% de variações em 527 casos; Dercutinis et al. (1987) ${ }^{9}$ avaliaram 253 doadores, com $27 \%$ de variações; Brems et al. (1989) $)^{10}$ descreveram 172 doadores, com $23 \%$ de variações; Todo et al. $(1987)^{11}$ obtiveram $35,5 \%$ de variações, em um estudo com 211 pacientes doadores cadáveres. Em nosso meio, Freitas (1997) ${ }^{12}$ realizou um estudo sobre a anatomia da artéria hepática em doadores e receptores de transplante hepático cadavérico, encontrando $24,36 \%$ de va- riações em 119 casos. Estes estudos enfatizavam a anatomia da origem da artéria hepática e de seus ramos direito e esquerdo. Em alguns destes estudos, troncos principais e acessórios não foram diferenciados ${ }^{7,10,11,12}$.

Em nosso estudo, considerando apenas os achados de cirurgia, houve $20 \%$ de variações na anatomia da artéria hepática. As variações diagnosticadas durante a captação de órgãos foram direcionadas, sendo que quando o lobo direito era captado, apenas a anatomia da artéria hepática direita era estudada em detalhes, inclusive em relação à presença de ramos acessórios e bifurcações extra-hepáticas. Em um dos nossos casos, a arteriografia foi normal, mas durante a cirurgia foi diagnosticado um ramo da artéria hepática comum direcionado ao setor posterior direito do fígado. Quando o lobo direito era captado, a anatomia da artéria hepática esquerda não era estudada, porém quando esta se apresentava evidente no ligamento gastro-hepático, era relatada como ramo da artéria gástrica esquerda. Situação semelhante ocorria quando o lobo esquerdo era captado: apenas a anatomia da artéria hepática

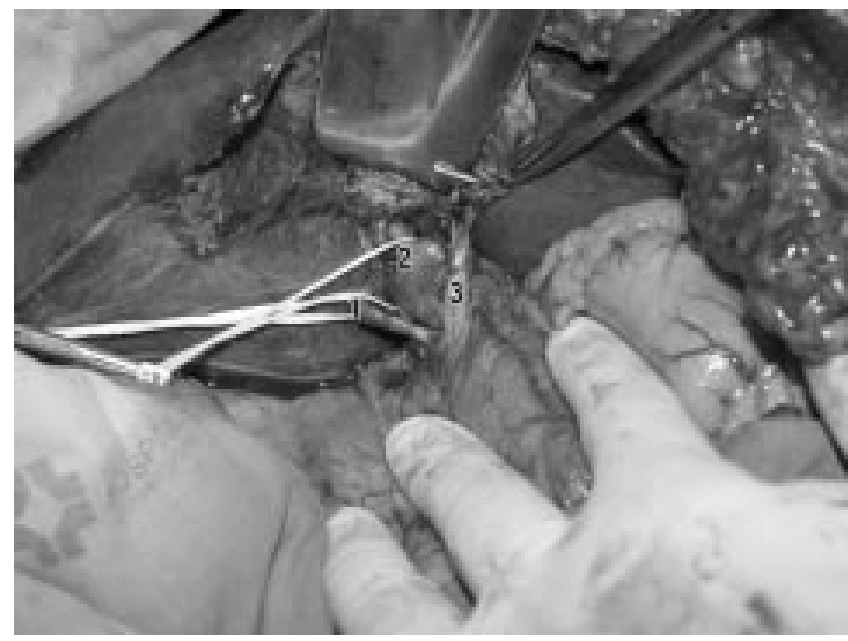

Figura 1 - Fotografia obtida no ato operatório de transplante hepático intervivos de um doador mostrando a artéria hepática direita ramo da artéria mesentérica superior. Nesta situação, a artéria hepática direita situa-se à direita do ducto colédoco.

Nota: 1 - Artéria hepática direita; 2 - veia porta; 3 - ducto colédoco. 
Tabela 3 - Comparação da anatomia da artéria hepática observada na arteriografia e na operação.

\begin{tabular}{rlll}
\hline N. ${ }^{0}$ Paciente & Receptor/Doador & Arteriografia & Cirurgia \\
\hline 51 & doador LD & AHD ramo AMS & AHD ramo AMS \\
81 & doador LD & AHD ramo AMS & AHD ramo AMS \\
79 & receptor & AHD ramo AMS & AHD ramo AMS \\
9 & doador LE & AHD ramo AMS & AHE ramo AGE, AHD ramo AMS \\
46 & doador LD & AHD acessória ramo AMS & AHD acessória ramo AMS \\
41 & doador LD & AHD acessória ramo AMS & AHD acessória ramo AMS \\
11 & doador LE & AHD acessória ramo AMS & AHE ramo TC, AHD acessória ramo AMS \\
3 & doador LE & AHD ramo da ACH, bifurcação precoce AHC & $\mathrm{n}$ \\
23 & doador LD & AHD ramo da AMS, AHE e ramo seg IV AHP & AHD ramo AMS \\
35 & doador LD & AHD ramoTC, AMS origina-se junto ao TC & AHD ramo TC \\
59 & doador LD & AHE bifurcada & $\mathrm{n}$ \\
74 & doador LD & AHE bifurcada & $\mathrm{n}$ \\
72 & doador LD & AHE bifurcada & $\mathrm{n}$ \\
75 & doador LD & AHE ramo TC e AHD ramo AMS & AHE ramo TC AHD ramo AMS \\
34 & doador LD & AHE ramo do TC & AHE ramo do TC \\
86 & doador LD & AHE ramo TC e AHD ramo AMS & $\mathrm{n}$ \\
56 & receptor & AHC ramo da AMS & AHC ramo AMS \\
61 & doador LD & normal & artéria setor posterior direito ramo AHP \\
20 & doador LE & TC e AMS se originam num tronco único & $\mathrm{n}$ \\
\hline
\end{tabular}

Nota: LD - lobo direito; LE - lobo esquerdo; AHD - artéria hepática direita; AHE - artéria hepática esquerda; AHC-artéria hepática comum; AMS - artéria mesentérica superior; TC - tronco celíaco; $n$ - não relatado.

esquerda era estudada minuciosamente. Entre os oito doadores de lobo esquerdo de nosso estudo, em dois casos a hepática esquerda foi diagnosticada como ramo da gástrica esquerda apenas durante a operação.

O estudo das arteriografias revelou uma taxa elevada $(41,86 \%)$ de variações da anatomia arterial hepática. Outros estudos de arteriografia mostraram resultados variáveis: Daly et al. ${ }^{13}$ obtiveram $30 \%$ de variações; Rygaard et al. ${ }^{14} 24,5 \%$ e Kemeny et al. ${ }^{15} 50 \%$.

Em relação a doadores vivos de fígado, além das variações do tronco da artéria hepática, também tem grande importância a identificação de todos os ramos arteriais no hilo hepático, inclusive os ramos acessórios e bifurcações extra-hepáticas das artérias hepáticas direita e esquerda. As reconstruções arteriais nos transplantes intervivos são mais complexas do que nos transplantes com fígado cadavérico, pois os ramos arteriais são mais finos e cur$\operatorname{tos}^{5,16}$

A duplicidade do suprimento arterial para determinado lobo ou segmento foi considerada uma contra-indicação relativa à doação intervivos ${ }^{17}$, pela necessidade de complexas reconstruções arteriais. Porém, a utilização de reconstruções microcirúrgicas propiciou a utilização de lobos hepáticos com mais de um ramo arterial $^{18}$, e atualmente poucos doadores potenciais são excluídos em razão de variações $\operatorname{arteriais}^{19,20}$.

A artéria hepática direita é em geral simples e, quando ramo da artéria mesentérica superior, facilita o procedimento de doação, pois o ramo arterial proveniente da artéria mesentérica superior é mais longo ${ }^{21}$. Em nosso estudo, três entre os 32 doadores de lobo direito possuíam artéria hepá- tica direita como ramo da artéria mesentérica superior $(9,37 \%)$; em dois outros casos, a artéria hepática direita foi ramo acessório da artéria mesentérica superior $(6,25 \%)$. No estudo de Nakamura et al. (2002) ${ }^{21}$, a artéria hepática direita foi ramo (principal ou acessório) da artéria mesentérica superior em $12,5 \%$ dos casos. Nos casos em que existiu um ramo acessório, Nakamura et $a l^{21}$. optaram pela ligadura do ramo acessório com reconstrução apenas do tronco principal. Em nosso estudo, não foi realizada dupla anastomose arterial em nenhum caso. Assim como ocorre no lobo direito, captação de lobos esquerdos com artérias hepáticas aberrantes (artéria hepática esquerda ramo da gástrica esquerda ou variações da artéria hepática comum), também gera enxertos com artérias de maior diâmetro e comprimento em relação a artérias hepáticas esquerdas normais ${ }^{22}$. Entre oito casos de doação do lobo hepático esquerdo, em dois a artéria hepática esquerda foi ramo da artéria gástrica esquerda (25\%). A artéria hepática esquerda normal possui uma taxa elevada de ramificação extra-hepática ${ }^{23}$. Em nosso estudo, três entre os 40 doadores $(7,5 \%)$ possuíam bifurcações extra-hepáticas da artéria hepática esquerda que foram diagnosticadas por arteriografia. Em todos esses casos, o lobo captado foi o direito.

É também importante a identificação da vascularização do segmento IV, que em geral é proveniente da artéria hepática esquerda, mas pode ser ramo da artéria hepática direita e que deve ser preservado na captação de lobos direitos ou segmentos laterais esquerdos.

Conclui-se que a prevalência de variações na anatomia da artéria hepática é elevada nos transplantes hepáticos intervivos. 


\begin{abstract}
Background: Detailed knowledge of hepatic artery anatomy is essential for the success of living related liver transplantation. The purpose of this study is to describe the anatomy of the hepatic artery of donors and recipients of living related liver transplantation. Methods: From March 1998 to September 2002, the study was retrospective (23 transplantations). From October 2002 to August 2003, the study was prospective (17 transplantations). We studied the hepatic artery anatomy of 80 consecutive patients (40 donors and 40 recipients) at the Liver Transplantation Unit of the Hospital de Clinicas of the Universidade Federal do Paraná and of the Hospital Nossa Senhora das Graças; 51 were male (27 recipients and 24 donors) and 29 female (13 recipients and 16 donors). The median age of the donors was 32.6 years and of the recipients was 36.3 years. Thirty-two recipients were adults and 8 were under than 15 years of age. Results: Forty-three patients underwent abdominal arteriography and variations of the hepatic artery anatomy were found in 18 of them (41.86\%). The most prevalent anomaly was the right hepatic artery arising from the superior mesenteric artery $(12.5 \% ; n=5)$. The anatomy of the hepatic artery was also evaluated at operation and variations were observed in 16 of the 80 patients (20\%). The most common variation observed at operation was the right hepatic artery arising from the superior mesenteric artery $(7.5 \% ; n=6)$. Conclusion: It is concluded that the prevalence of hepatic arterial variations is high.
\end{abstract}

Key Words: Liver transplantation; Living donors; Hepatic artery/anatomy \&histology.

\section{REFERÊNCIAS}

1. Raia S, Nery JR, Mies S. Liver transplantation from live donors. Lancet. 1989; 334(1): 497.

2. Strong RW, Lynch SV, Ong TH, et al. Successful liver transplantation from a liver donor to her son. N Engl J Med. 1990;322(6):1505-7.

3. Hashikura Y, Makuuchi M, Kawasaki, S, et al. Successful livingrelated partial liver transplantation to an adult patient. Lancet. 1994;343(2):1233-4.

4. Carone E, Chapchap P, Pugliese V, et al. Transplante hepático com doador vivo familiar: técnica operatória no doador. Rev Col Bras Cir. 1997; 24(4):235-40.

5. Desphande RR, Heaton ND, Rela M. Surgical anatomy of segmental liver transplantation. Br J Surg. 2002;89(3):1078-88.

6. Michels NA. Newer anatomy of the liver and its variant blood supply and collateral circulation. Am J Surg. 1966;112(4):33746.

7. Hiatt JR, Gabbay J, Busuttil RW. Surgical anatomy of the hepatic arteries in 1000 cases. Ann Surg, 1994;220(1): 50-2.

8. Soin AS, Friend PJ, Saxena R, et al. Donor arterial variations in liver transplantation: management and outcome of 527 consecutive grafts. Br J Surg 1996;83(4):637-41.

9. Decurtins JC, Friend PJ, Aranda H. Revascularization of liver grafts with recipient splenic artery in liver transplamtation. Transpl Proc. 1990;22(5):2376-7.

10. Bems JJ, Millis JM, Hiatt JR. Hepatic artery reconstruction during liver transplantation. Transplantation. 1989;47(3):403-6.

11. Todo S, Makowka L, Tzakis AG. Hepatic artery in liver transplantation. Transpl Proc. 1987;19(1):2406-11.

12. Freitas ACT. Anatomia da artéria hepática no transplante de fígado. Estudo de 119 órgãos no Hospital de Clínicas da Universidade Federal do Paraná. Curitiba, 1997. Dissertação (Mestrado) - Universidade Federal do Paraná.

13. Daly JM, Kenemy N, Oderman P. Long term hepatic infusion chemoterapy. Arch Surg 1984;119:936-41.

14. Rygaard H, Forrest M, Mygind T. Anatomic variants of the hepatic arteries. Acta Radiol Diag. 1986;27(6):425-7.

15. Kemeny MM, Hogan JM, Goldberg DA. Continuous hepatic artery infusion with an implantable pump: problems with hepatic artery anomalies. Surgery. 1993(4);113:395-402.
16. Ikegami T, Kawasaki S, Matsunami H, et al. Should all arterial branches be reconstruted in living- related liver transplantation? Surgery. 1996;119(4):431-6.

17. Broelsch CE, Emond JC, Whintington PF, et al. Application of reduced-size liver transplants as split grafts, auxiliary orthotopic grafts, and living related segmental transplants. Ann Surg 1990 ;212(3):368-77.

18. Mori K, Nagata I, Yamagata S. The introduction of microvascular surgery to hepatic artery reconstruction in living-donor liver transplantation- its surgical advantages compared with conventional procedures. Transplantation. 1992;54(3):263-8.

19. Marcos A, Killackey M, Orloff MS, et al. Hepatic arterial reconstruction in 95 adult right lobe living donor liver transplants: evolution of the anastomotic technique. Liver Transpl. 2003; 6(6):570-4.

20. Chen YS, Cheng YF, Villa VH, et al. Evaluation of living liver donors. Transplantation. 2003; 75(3):s16-9.

21. Nakamura T, Tanaka K, KiuchI T, et al. Anatomical variations and surgical strategies in right lobe living donor liver transplantation: lessons from 120 cases. Transplantation. 2002;73(12):1896-903.

22. Sakamoto Y, Takayama T, Nakatsuka T, et al. Advantage in using living donors with aberrant hepatic artery for partial liver graft arterialization. Transplantation. 2002;74(4):518-21.

23. Renz JF, Reichert PR, Emond JC. Biliary anatomy as applied to pediatric living donor and split-liver transplantation. Liver Transpl. 2000;6(6):801-4.

Como citar este artigo:

Soares RV, Coelho JCU, Matias JEF, Zeni Neto C, Freitas ACT, Godoy JL. Anatomia da artéria hepática em doadores e receptores de transplante hepático intervivos. Rev Col Bras Cir [periódico na Internet] 2006 Mar-Abr; 33(2). Disponível em URL: http:// www.scielo.br/rcbc

Endereço para correspondência:

Dr. Júlio Coelho

Rua Bento Viana, 1140 - Ap. 2202

80240-110 - Curitiba (PR)

Fone/Fax: (41) 3322-3789

E-mail: juliocoelho@bbs2.sul.com.br 